\title{
A scoping review of mental health mobile apps for use by the military community
}

\author{
Linna Tam-Seto, Valerie M. Wood, Brooke Linden, Heather Stuart \\ Health Services and Policy Research Institute, Queen's University, Kingston, ON, Canada \\ Contributions: (I) Conception and design: All authors; (II) Administrative support: B Linden, V Wood; (III) Provision of study material or patients: B \\ Linden, L Tam-Seto, V Wood; (IV) Collection and assembly of data: B Linden, L Tam-Seto, V Wood; (V) Data analysis and interpretation: L Tam- \\ Seto, V Wood; (VI) Manuscript writing: All authors; (VII) Final approval of manuscript: All authors. \\ Correspondence to: Linna Tam-Seto. Post-doctoral Fellow, Health Services and Policy Research Institute, Queen's University, Kingston, ON, Canada. \\ Email: linna.tam-seto@queensu.ca.
}

\begin{abstract}
Advancements in technology have opened doors to new directions in mental health care, including the emergence of mobile health applications. Such apps are helping to make mental health care more accessible to those who face barriers to care, such as military personnel. We conducted a scoping review to map the existing literature on mental health-related apps intended for use by military personnel/veterans. As a result, we identified several themes from the literature. We also discuss how apps are being developed and tested for use by the military community and provide suggestions for future research directions.
\end{abstract}

Keywords: Mental health; mobile applications; mhealth; military veterans; military personnel

Received: 13 October 2018; Accepted: 14 November 2018; Published: 12 December 2018.

doi: $10.21037 /$ mhealth.2018.12.01

View this article at: http://dx.doi.org/10.21037/mhealth.2018.12.01

\section{Introduction}

With the financial, social, and personal burden of mental and substance use disorders on the rise globally (1), the need for innovative, expansive, and accessible supports for mental health and wellness has perhaps never been greater. Fortunately, advancements in technology over the past decade have opened doors to interesting new directions in mental health care, moving away from person-delivered care towards more electronic or "e" delivered care. The spread and advancement of mobile technologies specifically has been a catalyst for such "eHealth" modalities, and has led to the emergence of "mHealth" (mobile health), the leverage of mobile devices to support medical or public health practices (2). One such functionality includes mobile software applications or "apps", which first became available for download in 2008 (3). By 2012, over 1.5 million apps had been developed with over 700 of these directly targeting mental health care (3). The ease of access to mobile applications regardless of location makes this an ideal resource for individuals who are relatively mobile and/ or isolated, such as members of the military community.
According to the National Defence and Canadian Forces Ombudsman's Special Report, "On the Homefront: Assessing the Well-being of Canada's Military Families in the New Millennium" (4), military life is characterized by mobility, separation, and risk. These life experiences can make accessing adequate health care challenging, and the need for accessible, innovative, and effective options paramount. In addition, military personnel face various barriers to accessing and seeking mental health care as a result of working in an environment that rewards physical and mental resilience, and where serious mental health concerns can place restrictions on a soldier's determined fitness for duty $(5,6)$.

Evidence suggests that there is a need for specialized mental health supports for military personnel. In 2013, one study found that within the Canadian Armed Forces (CAF), $32.2 \%$ of Regular Force personnel reported currently experiencing a mental health problem (7), with prevalence estimates for depression and generalized anxiety disorder almost doubling those observed among the general Canadian population (8). Despite the high prevalence of mental disorders among military personnel in both the 
United States (U.S.) and Canada, mental health services continue to be underutilized among this population (9).

A number of reviews have been conducted on the development of mHealth apps including an examination of evidence for their effectiveness (10), recommendations for their development (11) (Rickwood, \& Rickard, 2016) and apps that target specific mental health concerns such as posttraumatic stress disorder (PTSD) (12). However, no reviews have addressed the nature and scope of current literature on mHealth apps intended for use by military members, veterans, and their families.

\section{Methods}

We conducted a scoping review to map the existing literature on the availability of mental health-related mobile applications ("apps") intended for use by military personnel and/or veterans. Scoping reviews are an ideal method of increasing the understanding of the breadth and depth of literature on a specific topic that has not been previously examined (13). This study followed the five-step methodological framework developed by Arkey \& O'Malley (14) and further extended by Levac, Colquhoun, and O'Brien (15): (I) identification of the research question, (II) identification of relevant studies, (III) study selection (16), (IV) data extraction, and (V) content analysis.

\section{Identification of the research question}

Arksey and O'Malley (14) recommend formulating a broad enough research question to ensure a wide range of coverage, while limiting the possibility of missing pertinent articles. Levac, Colquhoun, and O'Brien (15) extend this strategy with their additional recommendations to combine this broad approach with a clearly articulated scope of inquiry, definition of concepts, identification of intended target populations, and outcomes of interest. With these goals in mind, we formulated the following research question: "What is currently documented in the research literature about mobile applications aimed at supporting the mental health of military members and veterans?" Specifically, we wanted to understand the purpose of existing apps, their coverage of mental health issues, the parameters of their development, and their core features.

\section{Identification of relevant studies and study selection}

One member of the research team searched four large academic databases based on recommendations from a reference librarian: PsycINFO, Cumulative Index of Nursing and Allied Health (CINAHL), Medical Literature Analysis and Retrieval System Online (Medline), and Excerpta Medica Database (Embase). Database searches were completed using key word combinations related to the following three inclusion criteria: mental health, military, and mobile application. Terms were searched in combination by key word and subject headings, using proximity searches and truncations to capture variations.

The research team developed and agreed upon the inclusion and exclusion criteria prior to the database search (15). Articles were screened into the study if they were of English-language, peer-reviewed quantitative or qualitative studies, and published between 2008 (the year apps were first available for download) and August 2018 (date of the review). We excluded records detailing computer-based applications that did not have a mobile app counterpart (see Figure 1).

\section{Data extraction and content analysis}

We reviewed articles using an analytic data guide that addressed the following: (I) general article information [including author(s), title, journal information, keywords, year of publication, research location, study design, and name of the mobile application described]; and (II) detailed information about the app discussed including its purpose (e.g., its mandate and intended audience), its core content (e.g., capabilities and features); its development (e.g., how the content was identified for use; development and testing procedures; parties involved); its feasibility; and study limitation/future directions. Two members of the research team screened articles into the review, examining them first by title, and then by abstract. Articles that were not relevant to the research question were removed from the sample, including, for example, scoping reviews and conference abstracts. Disagreements in inclusion decisions were resolved through discussion. NVivo12, qualitative data analysis software program (17) was used to assist in charting the data. This process-oriented method of data extraction is consistent with the approach recommended by Levac et al. (15).

\section{Results}

\section{General article information}

There were 35 papers included in the full text review (see 


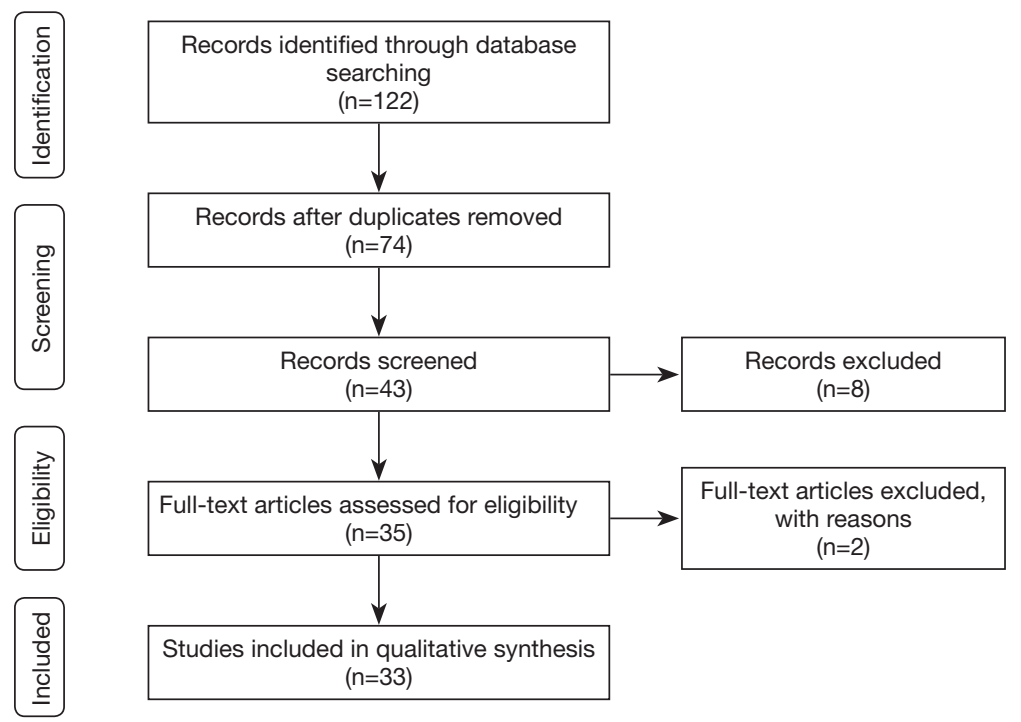

Figure 1 Flow diagram of article selection process.

Table 1 for a summary of article information). All of the articles were written by authors living in the U.S., however, two papers were the result of multi-country collaborations including one completed by authors from the U.S. and Israel (18) and another that included authors from the U.S., the Netherlands, Australia, Canada, Germany, Sweden, and Denmark (19). There were a range of study designs identified, with the most common being the use of randomized control trials. There were two systematic reviews included $(20,21)$ as well as four articles that were mobile app and/or program descriptions (19,22-24). Although the search parameters spanned 10 years, all of the included articles were published between 2012 and 2018. Sample sizes ranged from four [e.g., (16)] to 719 participants [e.g., (18)]. The population of interest in the articles included combat veterans, active military members, police officers, and clinicians working with military community members. We included police officers (25) as it frequently mentioned the relevance of military populations in the abstract and article body, including a description of the similarities between law enforcement and military populations, and the relevance of its mobile technology training program for military personnel.

\section{Information about the featured apps}

The mobile applications addressed a range of physical and mental health difficulties: sleep difficulties (16); smoking [We included smoking-related studies as they fell under the "addiction" umbrella search term and documented association with mental health (26)] (27,28); substance use disorder $(16,29)$; suicidal ideation (30,31); mood dysregulation (25,32-37); anger management (35,37-39); traumatic brain injury (40$42)$; and post-traumatic stress disorder $(18,19,23,24,43-49)$. We identified several themes in the literature regarding the utilization of mobile applications for members of the military community: (I) diverse range of mobile app's purpose, (II) evidence supporting the use of mobile apps, and (III) mobile apps are a part of a greater treatment program.

\section{Theme 1: diverse range of mobile app's purpose}

The purpose of the mobile apps described in the scoping review varied greatly and included a platform for enhanced therapeutic communication, a mobile version of established mental health interventions, a resource for information, a means to teach and practice specific coping skills or strategies, and to collect and communicate health information.

In several of the articles, the described mobile apps were developed to enhance existing therapeutic interactions between users and counsellors. For example, one of the features of the app described by Mackintosh et al. (38), the anger management treatment, is to support communication with a therapist. The mCare mobile app program $(35,50)$ is a bi-directional app whereby text messages are sent from the therapist to the user for communicating health tips, appointment reminders, and notifications for available 
Table 1 Summary of article information

\begin{tabular}{|c|c|}
\hline Article criteria & $\mathrm{N}$ (total $\mathrm{N}=35$ ) \\
\hline \multicolumn{2}{|l|}{ Author location } \\
\hline United States & 33 \\
\hline International collaborators & 2 \\
\hline \multicolumn{2}{|l|}{ Year of publication } \\
\hline 2012 & 2 \\
\hline 2013 & 4 \\
\hline 2014 & 9 \\
\hline 2015 & 6 \\
\hline 2016 & 6 \\
\hline 2017 & 5 \\
\hline 2018 & 3 \\
\hline \multicolumn{2}{|l|}{ Study design } \\
\hline Randomized control trial & 9 \\
\hline Mobile app/program description & 6 \\
\hline Cross-sectional & 4 \\
\hline Pilot study & 4 \\
\hline Program evaluation & 3 \\
\hline Field test & 2 \\
\hline Systematic review & 2 \\
\hline Proof of concept & 2 \\
\hline Cost-minimization & 1 \\
\hline Implementation study & 1 \\
\hline Case study & 1 \\
\hline Focus group & 1 \\
\hline \multicolumn{2}{|l|}{ Concern addressed } \\
\hline Post-traumatic stress disorder & 22 \\
\hline Mood dysregulation & 13 \\
\hline Sleep difficulties & 5 \\
\hline Traumatic brain injury & 5 \\
\hline Substance use disorder & 3 \\
\hline Anger management & 3 \\
\hline Smoking & 2 \\
\hline Suicidal ideation & 2 \\
\hline
\end{tabular}

Table 1 (continued)
Table 1 (continued)

\begin{tabular}{ll}
\hline Article criteria & $\mathrm{N}$ (total N=35) \\
\hline App utility/feasibility & 21 \\
Utility during treatment & 16 \\
General attitudes towards mobile app & 16 \\
Decrease in symptoms with use & 12 \\
Feasibility for future studies & 6 \\
Feedback on ease of use & 5 \\
Cost of utilization & 4 \\
Test-retest reliability of measures compared to & \\
paper version & 4 \\
Mobile app's ability to predict symptoms & 3 \\
Recommend to others & \\
\hline
\end{tabular}

updates. In addition, text messages are used to disseminate daily questionnaires that assess the service member's wellbeing, and expands the function of the app to include ongoing monitoring and evaluation. Two articles examined the utility of a counsellor-supported version of an existing mobile application $(44,45)$. For example, the effectiveness of the Clinical-supported PTSD Coach was compared to PTSD Coach, a self-management mobile app. In the CSPTSD Coach, there is clinician support offered through the app to provide brief intervention (Possamato, Kuh, Johnson, Hoffman, etc.), and give additional structure, and guidance. Such tailoring is intended to encourage use of the mobile app.

Some mobile apps are used to deliver a mobile version of a specific therapeutic intervention. The Cognitive Behavioral Therapy for Insomnia (CBT-I) mobile app was explored as a portable alternative to provide cessation therapy for veterans with cannabis use disorders as well and sleep difficulties (16). Babson et al. (16) demonstrated how CBT-I showed promising results in providing clinical benefits for individuals with alcohol dependence. Another therapeutic intervention that has been shown to be beneficial for military members struggling with suicidal thoughts is the use of a Hope Box $(30,31)$. The purpose of a Hope Box is "to provide a means of recalling (an individual's) reasons for living during periods of significant distress and discouragement when they may be susceptive 
to suicidal thinking" (30). The personal reminders initiated by Hope Box have been shown to be useful in distracting individuals from their suicidal thoughts and with the ultimate goal of increasing general stress tolerance skills $(30,31)$. However, there are practical and logical challenges with carrying around a physical box, which inspired researchers to explore a virtual version.

The literature also highlights mobile apps that were developed to teach specific skills used to self-manage mental health symptoms. Breath2Relax (41) is described as a mobile app to teach diaphragmatic breathing to support psychological well-being and symptoms of traumatic brain injuries in members of the military community. Another virtual tool designed to support the development of psychologically adaptive skills and strategies is the Personal Health Intervention (PHIT) Toolkit for Duty $(22,51)$. This evidence-based mobile application consists of mindfulnessbased relaxation training and behavioural education in sleep quality and alcohol use (51).

Mobile apps provide an effective and convenient opportunity to collect health information to support the ability of the user to self-manage or to store information to share with health care provider during future appointments. T2 Mood Tracker (32) and AccWalker (42) are examples of mobile applications that collect information that can be used for additional clinical and research purposes. Although they differ with respect to the type of information collected (the T2 Mood Tracker detects shifts in the military member's mood, while the AccWalker captures changes in neuromotor functioning in response to low-level blast exposure), the utilization of such mobile apps demonstrates the range of functionalities that are currently being explored.

\section{Theme 2: evidence supporting use of mobile apps}

Of the articles that examined utility or feasibility of a mobile app $(\mathrm{n}=31)$, all reported that the mobile apps were useful. Utility and feasibility were examined from various perspectives including: appropriateness for further feasibility studies [e.g., (16)]; quality feedback on ease of use [e.g., (30,32)]; test-retest reliability when compared to paper versions $(33,51)$; utility during treatment $(23,27,29,31,44,49,50)$; general attitudes toward the mobile app [e.g., $(35,40,43,45,46)]$; overall financial cost of utilization [e.g., $(18,41)$ ]; predicting symptoms and/or changes $(36,42)$; and decrease in self-reported and actual symptoms [e.g., (25,34,37-39,47)].

The proof-of-concept study of the Virtual Hope Box
(VHB) (30) determined that users overwhelmingly found the mobile app to be beneficial and helpful. Ease of set up and use were important aspects of the mobile app. Results from this study also highlighted that users were also likely to use the app as well as recommend it to others.

Support for mobile apps was also measured by decreased self-reported mental health symptoms such as decreased tension, irritability, anxiety, depression (34), anger (38), and those related to PTSD $(39,47,49)$.

Costs incurred by the user was an observed index of utility and feasibility. In fact, the purpose of the study by Luxton et al. (41) was to evaluate the projected cost of care through use of the app relative to in-person care (i.e., cost-minimization analysis). They concluded that there was potential for a substantial societal savings in health care costs with a large number of users, suggesting that wide spread use of mobile apps could be economically beneficial at the societal level. Another study examined the use of attention bias modification training (ABMT), a computer-based protocol to help train infantry soldiers to shift attention away from threat thus decreasing anxiety in theatre, indicated the program's low cost and high scalability potential (18).

Access to mobile devices as well as attitudes towards the utility of mobile apps were also considered when determining overall feasibility. In a study by Erbes and colleagues (48), the authors explored the access, utilization, and interest in existing mobile applications for U.S. veterans receiving outpatient services for PTSD. They found that although a large majority $(76 \%)$ of respondents had access to mobile and tablet devices, only, $10 \%$ of those participants had used a mobile app. This study also found that age of the user was a significant predictor of device ownership but not of utilization or interest in the use of mobile apps. Overall, individuals that had access to devices were more likely to be interested in, or currently using, mobile apps regardless of age.

\section{Theme 3: mobile apps a part of a greater treatment program}

Some mobile apps included in this review were described as playing a role in supporting larger, multi-module programs, treatments, or interventions that focused on the mental health and well-being of military members or veterans. For example, a study by Shaw and colleagues (29), provided veterans enrolled in an outpatient treatment program for alcohol and substance abuse recovery with iPods containing various recovery-related podcasts and 
mobile apps. The purpose of this study was to determine whether or not the utilization of portable resources would provide complementary education and support between each outpatient session (29). Based on pretest and post-test surveys, this descriptive pilot study indicated that veterans attending outpatient treatment for alcohol and substance abuse found using mobile devices effective in augmenting their current treatment plan and was useful in their recovery. Similar success was found in the outcomes of a study aimed at promoting cognitive support technology use and employment success for postsecondary students living with traumatic brain injuries (40). This multi-year, multisite initiative entitled Project Career aimed to combine technology with vocational rehabilitation in both civilian and veteran participants. The participants of the study reported that using the mobile app improved their mood, and made them more independent and social.

The Stay Quit Coach mobile app was examined for utility within an integrated care protocol, an 8-week PTSD-informed cognitive behavioural therapy program for smoking cessation aimed at supporting veterans (28). Other components of the integrated care protocol included psychopharmacological and monthly follow-up therapy sessions. Stay Quit Coach was developed to provide evidence-based strategies to manage smoking urges and PTSD symptoms including personalized coping plans to manage stress and mood changes, proactive alerts, and motivational messages.

\section{Discussion}

The purpose of this scoping review was to determine what is documented in the research literature about mobile applications aimed at supporting the mental health of military community members. The results of this study revealed some interesting commonalities in this nascent research area.

Many of the studies reviewed were aimed at determining whether or not mobile apps are perceived to be an acceptable form of mental health support by members of the military community, through either an assessment of users' general attitudes of the app, perceived ease of use, and whether they would recommend the app to others. Overwhelmingly, the studies determined that there was a general willingness to use apps, likely because of the increased acceptability of this platform for resources and support. This acceptance is reflected in a global increase in the use of mobile devices. Currently, over $85 \%$ of the world's population is covered by a wireless signal. In the U.S. in particular, it is estimated that over $95 \%$ of Americans own a cell phone, with $77 \%$ of those owning a smartphone (52).

More importantly, it appears that military members perceive mobile apps to be an ideal supplement to traditional mental health care because they can adequately address issues with underutilization of mental health services. In a 2013 epidemiological survey investigating the mental health of CAF personnel, about $15 \%$ of Regular Force personnel reported experiencing a need for mental health care in the past 12 months, but not receiving it (7). The survey identified that the most commonly cited reasons for not receiving mental health care were a preference for self-management (64\%), fear that seeking help would negatively affect one's career (39\%), and fear of asking for help (28\%). This is an indication that many Regular Force personnel perceive there to be a substantial degree of stigma associated with mental illness, in addition to perceived structural barriers to care and career implications, potentially explaining the underutilization of mental health care within this population (9).

The use of mobile technologies for mental health support and care may be a desirable option for many military members and veterans who fear stigmatization and the career implications of seeking help. Mobile apps in particular offer a solution given the potential for greater confidentiality, addressing stigma-related barriers including career implications and social judgment, and meeting the reported need of Regular Force members for selfmanagement of mental health concerns (7). In addition to the stigma-related and structural barriers already mentioned, many CAF Regular Force personnel reported occupational or social barriers to care such as scheduling issues, or being in training or deployed at the time of need. By providing care that is accessible almost anywhere and anytime, mobile apps can address many of these identified barriers. Therefore, such solutions may entice many who are currently not seeking care due to perceived barriers or preferences for self-management to do so, leading to early identification and intervention for mental health-related concerns, and ultimately reducing the burden of mental health problems at the population level.

Clearly, the number of mobile apps documented for use by military populations specifically in the academic literature is limited in number, scope, and location. Further international collaboration and research in the development and testing of mobile apps for military community members is necessary. The parameters established by this particular 
scoping review only identified those mobile apps described in peer-reviewed journals, which all happened to be based out of the U.S.. However, there are emerging initiatives occurring internationally. In Canada, the CAF has developed the "R2MR" (Road to Mental Readiness) mobile app that provides military members access to content from its in-class R2MR deployment training program, and meant to improve skills related to mental health including goalsetting, self-talk, tactical breathing, and attention control. In addition, \#Here4U (military version) is a mobile app currently under development in Canada (53), a result of a collaboration between academia and industry. The purpose of \#Here $4 U$ is to provide personalized mental health supports to members of the military community, including CAF personnel, veterans, and adult family members.

In addition to this review highlighting a need for international collaboration and inclusion of international samples, future research should continue to focus on the validation of mobile apps developed for, or used by, military populations using evidence-based approaches. Indeed, in our review only nine articles involved the use of randomized control trials to test the efficacy of mobile apps to improve the mental well-being of military members or veterans. In addition to being one of the non-American-based mobile apps, \#Here4U (military version) is being developed to ensure that the app itself is evidence-based and will undergo rigorous testing (53). At this time, currently available mobile apps are relying on the users to determine for themselves the clinical effectiveness of the mobile apps they are using.

As we have outlined, the military community faces unique life experiences that can make access to adequate health care challenging. Leveraging the use of mobile technologies promises to be an exciting, innovative, and effective solution to making mental health support more accessible for military members, veterans, and their families.

\section{Limitations}

This scoping review did not follow a citation trail, which may have limited the articles that were included in this study. Search terms used in the database search were not standardized. The findings of this study may have been enhanced through the inclusion of broadened database across health care and technology-based fields.

\section{Conclusions}

The purpose of this study was to determine what is currently documented in the research literature about mobile applications aimed at supporting the mental health of military members and veterans. Using a scoping review method, several themes were identified including discussion about the diverse range of mobile app's purpose, descriptions of evidence supporting the use of mobile apps, and utilization of mobile apps are a part of a greater treatment program. The results of this study provide us with some understanding to how mobile apps are being developed and used with members of the military community, while also helping identify gaps and provide guidance for future directions. As research continues in this area, meta-analyzes can be conducted to determine effectiveness of mhealth mobile apps with this population. Also, with the expanded global use of mobile apps may encourage increased international research to reflect the utilization of new technology. Overall, the use of mobile apps to support the health and well-being of military community members shows promising prospects.

\section{Acknowledgements}

None.

\section{Footnote}

Conflicts of Interest: The authors have no conflicts of interest to declare.

\section{References}

1. Whiteford HA, Degenhardt L, Rehm J, et al. Global burden of disease attributable to mental and substance use disorders: findings from the Global Burden of Disease Study 2010. Lancet 2013;382:1575-86.

2. Kay M, Santos J, Takane M. MHealth: New horizons for health through mobile technologies. In: Global Observatory for E Health Series: Volume 3. World Health Organization, Switzerland. 2011. Available online: http:// www.who.int/goe/publications/goe_mhealth_web.pdf

3. News MH. Report: $13 \mathrm{~K}$ iPhone consumer health apps in 2012. 2012. Available online: https://www. mobihealthnews.com/13368/report-13k-iphoneconsumer-health-apps-in-2012

4. Ombudsman Department of Nation Defence and Canadian Forces. On the homefront: Assessing the wellbeing of Canada's military families in the new millennium. In: Forces NDaC. editor. Ottawa, ON: Office of the 
Ombudsman, 2013.

5. Nash WP, Silva C, Litz B. The historic origins of military and veteran mental health stigma and the stress injury model as a means to reduce it. Psychiatr Ann 2009;39:789-94.

6. Convoy S, Westphal RJ. The importance of developing military cultural competence. J Emerg Nurs 2013;39:591-4.

7. Theriault F, Gabler K, Naicker K. Health and lifestyle information survey of Canadian Forces personnel 2013/2014 - Regular Force report. Ottawa, ON: Department of National Defence,2016. Contract No.: D2293/2010E-PDF.

8. Pearson C, Zamorski M, Janz T. Mental health of the Canadian Armed Forces. 2014. Accessed January 3, 2016. Available online: http://www.statcan.gc.ca/pub/82624-x/2014001/article/14121-eng.htm

9. Fikretoglu D, D'Agata MT, Sullivan-Kwantes W, et al. Mental Health and Mental Health Service Use Attitudes among Canadian Armed Forces (CAF) Recruits and Officer Cadets. Ottawa, ON: Defence Research and Development Canada, 2017. Contract No.: DRDCRDDC-2017-R027.

10. Donker T, Petrie K, Proudfoot J, et al. Smartphones for smarter delivery of mental health programs: a systematic review. J Med Internet Res 2013;15:e247.

11. Bakker D, Kazantzis N, Rickwood D, et al. Mental Health Smartphone Apps: Review and Evidence-Based Recommendations for Future Developments. JMIR Ment Health 2016;3:e7.

12. Rodriguez-Paras C, Tippey K, Brown E, et al. Posttraumatic Stress Disorder and Mobile Health: App Investigation and Scoping Literature Review. JMIR Mhealth Uhealth 2017;5:e156.

13. Mays N, Roberts E, Popay J. Synthesising reserach evidence. Studying the organisation and delivery of health services: Research methods. London: Routledge, 2001.

14. Arksey H, O'Malley L. Scoping studies: Towards a methodological framework. Int J Soc Res Methodol 2005;8:19-32.

15. Levac D, Colquhoun H, O'Brien KK. Scoping studies: advancing the methodology. Implement Sci 2010;5:69.

16. Babson KA, Ramo DE, Baldini L, et al. Mobile AppDelivered Cognitive Behavioral Therapy for Insomnia: Feasibility and Initial Efficacy Among Veterans With Cannabis Use Disorders. JMIR Res Protoc 2015;4:e87.

17. QSR International Pty Ltd. NVivo qualitative data analysis Software. Melbourne, Australia, 2018.

18. Wald I, Fruchter E, Ginat K, et al. Selective prevention of combat-related post-traumatic stress disorder using attention bias modification training: a randomized controlled trial. Psychol Med 2016;46:2627-36.

19. Kuhn E, van der Meer C, Owen JE, et al. PTSD Coach around the world. Mhealth 2018;4:15.

20. Dedert E, Williams JW, Stein R, et al. Evidence Report: E-Interventions for Alcohol Misuse. Washington, DC: Department of Veterans Affairs, 2014. Contract No.: \#09-010.

21. Dyer EA, Kansagara D, McInnes DK, et al. Mobile Applications and Internet-based Approaches for Supporting Non-professional Caregivers: A Systematic Review. Washington, DC: Department of Veterans Affairs, 2012. Contract No.: \#05-225.

22. Kizakevich PN, Hubal R, Brown J, et al. PHIT for duty, a mobile approach for psychological health intervention. Stud Health Technol Inform 2012;181:268-72.

23. Reger GM, Hoffman J, Riggs D, et al. The "PE coach" smartphone application: an innovative approach to improving implementation, fidelity, and homework adherence during prolonged exposure. Psychol Serv 2013;10:342-9.

24. Xu R, Mei G, Zhang G, et al. TPM: cloud-based telePTSD monitor using multi-dimensional information. Stud Health Technol Inform 2013;184:471-7.

25. Weltman G, Lamon J, Freedy E, et al. Police department personnel stress resilience training: an institutional case study. Glob Adv Health Med 2014;3:72-9.

26. Burki TK. Smoking and mental health. Lancet Respir Med 2016;4:437.

27. Carpenter VL, Hertzberg JS, Kirby AC, et al. Multicomponent smoking cessation treatment including mobile contingency management in homeless veterans. J Clin Psychiatry 2015;76:959-64.

28. Herbst E, Pennington D, Kuhn E, et al. Mobile Technology for Treatment Augmentation in Veteran Smokers With Posttraumatic Stress Disorder. Am J Prev Med 2018;54:124-8.

29. Shaw BR, Sivakumar G, Balinas T, et al. Testing the Feasibility of Mobile Audio-Based Recovery Material as an Adjunct to Intensive Outpatient Treatment for Veterans with Substance Abuse Disorders. J Technol Hum Serv 2013;31:321-36.

30. Bush NE, Dobscha SK, Crumpton R, et al. A Virtual Hope Box smartphone app as an accessory to therapy: proof-of-concept in a clinical sample of veterans. Suicide Life Threat Behav 2015;45:1-9.

31. Bush NE, Smolenski DJ, Denneson LM, et al. A Virtual 
Hope Box: Randomized Controlled Trial of a Smartphone App for Emotional Regulation and Coping With Distress. Psychiatr Serv 2017;68:330-6.

32. Bush NE, Ouellette G, Kinn J. Utility of the T2 Mood Tracker mobile application among army warrior transition unit service members. Mil Med 2014;179:1453-7.

33. Bush NE, Skopp N, Smolenski D, et al. Behavioral screening measures delivered with a smartphone app: psychometric properties and user preference. J Nerv Ment Dis 2013;201:991-5.

34. Kahn JR, Collinge W, Soltysik R. Post-9/11 Veterans and Their Partners Improve Mental Health Outcomes with a Self-directed Mobile and Web-based Wellness Training Program: A Randomized Controlled Trial. J Med Internet Res 2016;18:e255.

35. Pavliscsak H, Little JR, Poropatich RK, et al. Assessment of patient engagement with a mobile application among service members in transition. J Am Med Inform Assoc 2016;23:110-8.

36. Place S, Blanch-Hartigan D, Rubin C, et al. Behavioral Indicators on a Mobile Sensing Platform Predict Clinically Validated Psychiatric Symptoms of Mood and Anxiety Disorders. J Med Internet Res 2017;19:e75.

37. Winslow BD, Chadderdon GL, Dechmerowski SJ, et al. Development and Clinical Evaluation of an mHealth Application for Stress Management. Front Psychiatry 2016;7:130.

38. Mackintosh MA, Niehaus J, Taft CT, et al. Using a Mobile Application in the Treatment of Dysregulated Anger Among Veterans. Mil Med 2017;182:e1941-9.

39. Morland LA, Niehaus J, Taft C, et al. Using a Mobile Application in the Management of Anger Problems Among Veterans: A Pilot Study. Mil Med 2016;181:990-5.

40. Hendricks DJ, Sampson E, Rumrill P, et al. Activities and interim outcomes of a multi-site development project to promote cognitive support technology use and employment success among postsecondary students with traumatic brain injuries. NeuroRehabilitation 2015;37:449-58.

41. Luxton DD, Hansen RN, Stanfill K. Mobile app selfcare versus in-office care for stress reduction: a cost minimization analysis. J Telemed Telecare 2014;20:431-5.

42. Rhea CK, Kuznetsov NA, Ross SE, et al. Development of a Portable Tool for Screening Neuromotor Sequelae From Repetitive Low-Level Blast Exposure. Mil Med 2017;182:147-54.

43. Kuhn E, Greene C, Hoffman J, et al. Preliminary evaluation of PTSD Coach, a smartphone app for posttraumatic stress symptoms. Mil Med 2014;179:12-8.

44. Possemato K, Kuhn E, Johnson E, et al. Using PTSD Coach in primary care with and without clinician support: a pilot randomized controlled trial. Gen Hosp Psychiatry 2016;38:94-8.

45. Possemato K, Kuhn E, Johnson EM, et al. Development and refinement of a clinician intervention to facilitate primary care patient use of the PTSD Coach app. Transl Behav Med 2017;7:116-26.

46. Reger GM, Skopp NA, Edwards-Stewart A, et al. Comparison of Prolonged Exposure (PE) Coach to Treatment as Usual: A Case Series With Two Active Duty Soldiers. Mil Psychol 2017;27:287-96.

47. Roy MJ, Costanzo ME, Highland KB, et al. An App a Day Keeps the Doctor Away: Guided Education and Training via Smartphones in Subthreshold Post Traumatic Stress Disorder. Cyberpsychol Behav Soc Netw 2017;20:470-8.

48. Erbes CR, Stinson R, Kuhn E, et al. Access, utilization, and interest in mHealth applications among veterans receiving outpatient care for PTSD. Mil Med 2014;179:1218-22.

49. Roy MJ, Highland KB, Costanzo MA. GETSmart: Guided Education and Training via Smart Phones to Promote Resilience. Stud Health Technol Inform 2015;219:123-8.

50. Poropatich RK, Pavliscsak HH, Tong JC, et al. mCare: using secure mobile technology to support soldier reintegration and rehabilitation. Telemed J E Health 2014;20:563-9.

51. Kizakevich PN, Eckhoff R, Brown J, et al. PHIT for Duty, a Mobile Application for Stress Reduction, Sleep Improvement, and Alcohol Moderation. Mil Med 2018;183:353-63.

52. Centre PR. Mobile Technology Fact Sheet. Available online: 2018. http://www.pewinternet.org/fact-sheet/ mobile/

53. Wood VM, Linden B, Tam-Seto L, et al. Military Personnel, Families, and Veterans, We Are \#Here4U: Introducing our E-Solution for Military Mental Wellness. Poster presentation at the Canadian Academy of Canadian Epidemiology 2018 Annual Scientific Symposium, Toronto, ON. 2018.

doi: $10.21037 /$ mhealth.2018.12.01

Cite this article as: Tam-Seto L, Wood VM, Linden B, Stuart H. A scoping review of mental health mobile apps for use by the military community. mHealth 2018;4:57. 